\title{
Pd/Cu-Catalyzed Dehydrogenative Coupling of Dimethyl Phthalate: Synchrotron Radiation Sheds Light on the Cu Cycle Mechanism
}

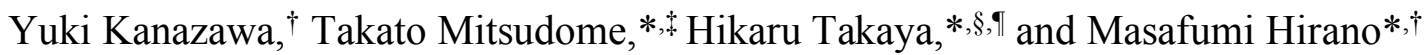 \\ †Department of Applied Chemistry, Graduate School of Engineering, Tokyo University of Agriculture and Technology, 2- \\ 24-16 Nakacho, Koganei, Tokyo 184-8588, Japan \\ *Department of Materials Engineering Science, Graduate School of Engineering Science, Osaka University, 1-3 \\ Machikaneyama, Toyonaka, Osaka 560-8531, Japan \\ §Institute of Chemical Research, Kyoto University, Gokasho, Uji, Kyoto 611-0011, Japan \\ Institute for Molecular Research, National Institutes of Natural Science, Nishigo-Naka, Myodaiji, Okazaki 444-8585, Japan
}

\section{Corresponding Authors}

*E-mail for M.H.: hrc@cc.tuat.ac.jp.

*E-mail for T.M.: mitsudom@cheng.es.osaka-u.ac.jp.

*E-mail for H.T.: takaya@scl.kyoto-u.ac.jp. 


\section{Table of Contents}

${ }^{1} \mathrm{H}$ NMR Spectrum of $\left[\mathrm{Pd}(\mathrm{OAc})_{2}(\right.$ phen $\left.)\right](\mathbf{1 b})$ S3

${ }^{13} \mathrm{C}\left\{{ }^{1} \mathrm{H}\right\}$ NMR Spectrum of $\left[\mathrm{Pd}(\mathrm{OAc})_{2}\right.$ (phen) $](\mathbf{1 b})$

Molecular structure of $\left[\mathrm{Cu}(\mathrm{OAc})_{2}\right]_{2} \cdot 2 \mathrm{AcOH}(2 \mathbf{a} \cdot \mathrm{AcOH})$

Crystallographic data for $\left[\mathrm{Cu}(\mathrm{OAc})_{2}\right]_{2} \cdot 2 \mathrm{AcOH}(2 \mathrm{a} \cdot \mathrm{AcOH})$

Atomic coordinates for $\left[\mathrm{Cu}(\mathrm{OAc})_{2}\right]_{2} \cdot 2 \mathrm{AcOH}(2 \mathrm{a} \cdot \mathrm{AcOH})$

S6

Anisotropic parameters for $\left[\mathrm{Cu}(\mathrm{OAc})_{2}\right]_{2} \cdot 2 \mathrm{AcOH}(2 \mathbf{a} \cdot \mathrm{AcOH})$

S7

Bond distances for $\left[\mathrm{Cu}(\mathrm{OAc})_{2}\right]_{2} \cdot 2 \mathrm{AcOH}(2 \mathrm{a} \cdot \mathrm{AcOH})$

S8

Bond angles for $\left[\mathrm{Cu}(\mathrm{OAc})_{2}\right]_{2} \cdot 2 \mathrm{AcOH}(2 \mathrm{a} \cdot \mathrm{AcOH})$

59

Dihedral angles for $\left[\mathrm{Cu}(\mathrm{OAc})_{2}\right]_{2} \cdot 2 \mathrm{AcOH}(2 \mathbf{a} \cdot \mathrm{AcOH})$

FEFF fitting analysis of $\mathrm{Cu}$ species in the reaction mixture of $\left[\mathrm{Pd}(\mathrm{OAc})_{2}(\right.$ phen $\left.)\right](1 \mathbf{b}) / \mathrm{Cu}(\mathrm{OAc})_{2} \cdot \mathrm{H}_{2} \mathrm{O}\left(\mathbf{2} \mathbf{a} \cdot \mathrm{H}_{2} \mathrm{O}\right) \mathrm{S} 11$ 


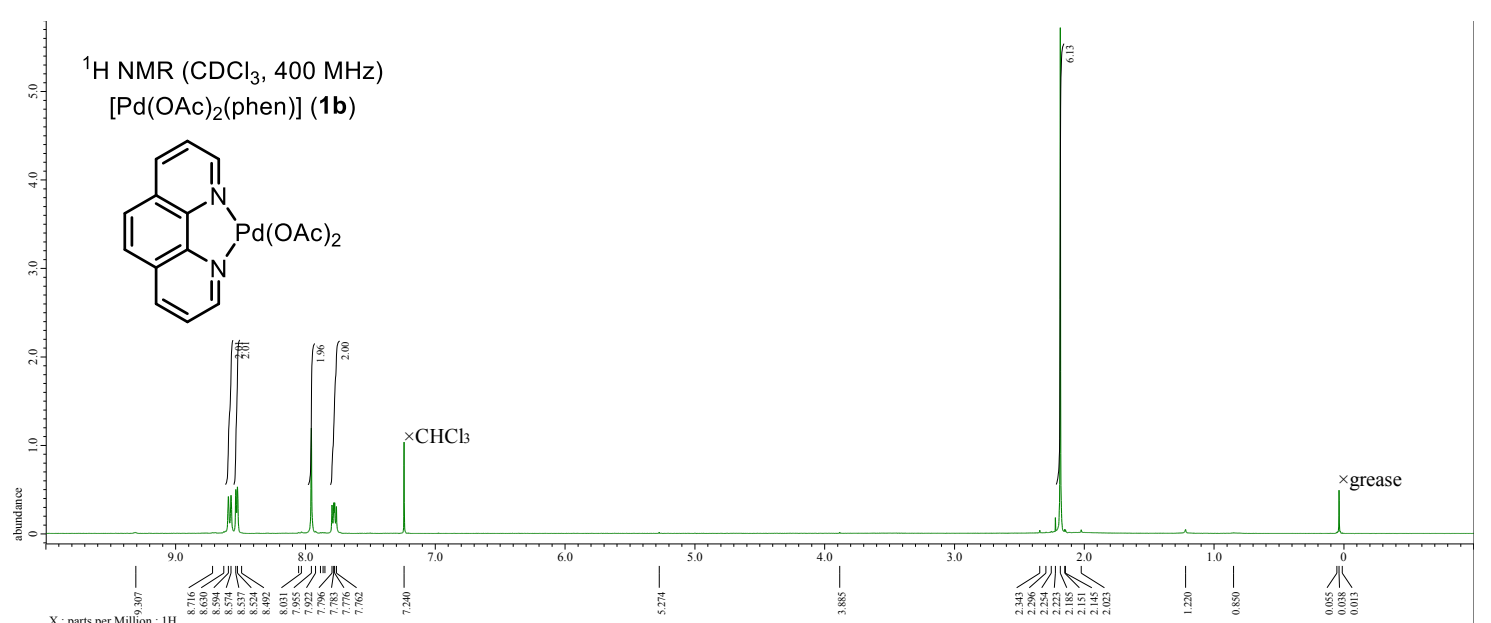

Figure S1. ${ }^{1} \mathrm{H}$ NMR spectrum of $\left[\mathrm{Pd}(\mathrm{OAc})_{2}(\right.$ phen $\left.)\right]($ 1b) generated by the stoichiometric reaction of $[\mathrm{Pd}(\mathrm{dba})($ phen $)]$ (7) with $\mathrm{Cu}(\mathrm{OAc})_{2} \cdot \mathrm{H}_{2} \mathrm{O}\left(2 \mathrm{a} \cdot \mathrm{H}_{2} \mathrm{O}\right)\left(400 \mathrm{MHz}, \mathrm{CDCl}_{3}\right)$.

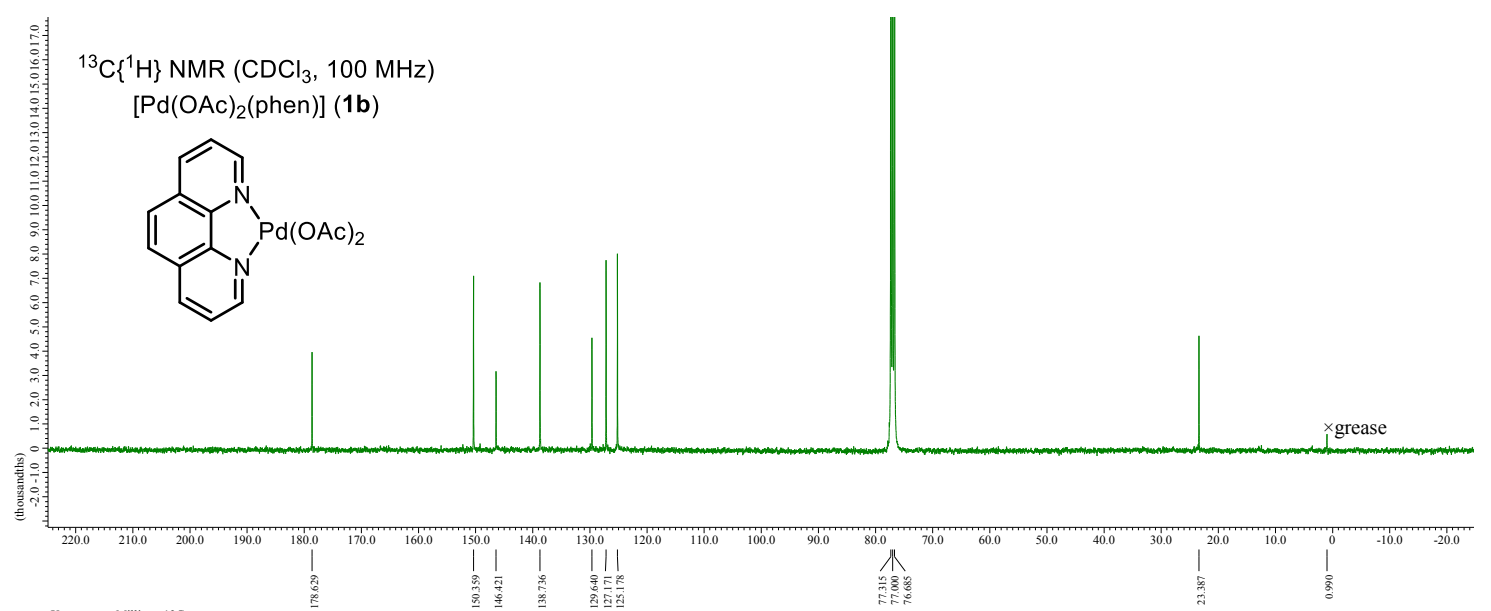

Figure $\mathbf{S 2} .{ }^{13} \mathrm{C}\left\{{ }^{1} \mathrm{H}\right\}$ NMR spectrum of $\mathbf{1} \mathbf{b}$ generated by the stoichiometric reaction of $\mathbf{7}$ with $\mathbf{2 a} \cdot \mathrm{H}_{2} \mathrm{O}(100 \mathrm{MHz}$, $\left.\mathrm{CDCl}_{3}\right)$. 


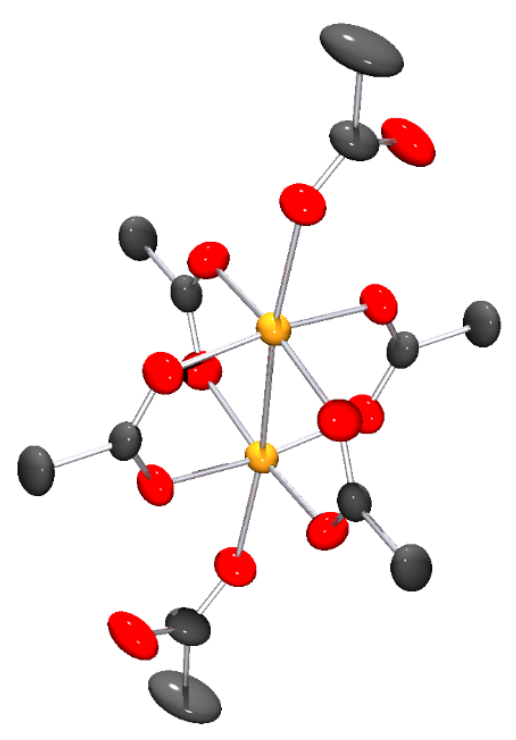

Figure S3. Molecular structure of a dimer of $\left[\mathrm{Cu}(\mathrm{OAc})_{2}\right] 2 \cdot 2 \mathrm{AcOH}(2 \mathrm{a} \cdot \mathrm{AcOH})$ by $\mathrm{X}$-ray structure analysis. All hydrogen atoms are omitted for clarity. Ellipsoids represent $50 \%$ probability. 
Table S1. Crystallographic Data for $\left[\mathrm{Cu}(\mathrm{OAc})_{2}\right]_{2} \cdot 2 \mathrm{AcOH}(2 \mathrm{a} \cdot \mathrm{AcOH})$.

\begin{tabular}{|c|c|}
\hline chemical formula & $\mathrm{C}_{6} \mathrm{H}_{10} \mathrm{CuO}_{6}$ \\
\hline formula weight & 241.69 \\
\hline crystal system & monoclinic \\
\hline space group & $P 21 / n$ (No. 14$)$ \\
\hline$a(\AA)$ & $8.228(6)$ \\
\hline$b(\AA)$ & $7.776(5)$ \\
\hline$a(\AA)$ & $15.160(11)$ \\
\hline$\beta(\operatorname{deg})$ & $102.855(7)$ \\
\hline$V\left(\AA^{3}\right)$ & $945.6(11)$ \\
\hline Z & 4 \\
\hline measurement reflns used & 3139 \\
\hline crystal description & prism \\
\hline crystal color & blue \\
\hline crystal size (mm) & $0.466 \times 0.363 \times 0.288$ \\
\hline crystal density diffrn $\left(\mathrm{g} \mathrm{cm}^{-3}\right)$ & 1.697 \\
\hline$F(000)$ & 492.00 \\
\hline$\mu\left(\mathrm{cm}^{-1}\right)$ & 2.305 \\
\hline measured temp $(\mathrm{K})$ & 293 \\
\hline radiation type & Mo K $\alpha(\lambda=0.71075 \AA)$ \\
\hline measurement device & Rigaku Mercury 70 \\
\hline measurement method & $\omega$ \\
\hline reflns numbers & 6615 \\
\hline reflns number total & 2155 \\
\hline reflns number gt & 2011 \\
\hline reflns threshold expression & $F^{2}>2.0 \sigma\left(F^{2}\right)$ \\
\hline$R$ & 0.0473 \\
\hline$w R$ & 0.1227 \\
\hline refine Is number reflns & 2155 \\
\hline refine Is number parameters & 122 \\
\hline GOF & 1.128 \\
\hline
\end{tabular}


Table S2. Atomic Coordinates for $\left[\mathrm{Cu}(\mathrm{OAc})_{2}\right]_{2} \cdot 2 \mathrm{AcOH}(\mathbf{2 a} \cdot \mathrm{AcOH})$.

\begin{tabular}{lllll}
\hline atom & $x$ & $y$ & $z$ & $U_{\text {ani }}$ \\
\hline Cu1 & $0.97000(3)$ & $0.08112(3)$ & $0.56890(2)$ & $0.02847(16)$ \\
O1 & $0.7634(2)$ & $-0.0524(3)$ & $0.54499(16)$ & $0.0434(4)$ \\
O2 & $0.8148(2)$ & $-0.1914(2)$ & $0.42521(14)$ & $0.0422(4)$ \\
O3 & $1.0841(3)$ & $-0.1053(2)$ & $0.64566(13)$ & $0.0431(4)$ \\
O4 & $1.1356(2)$ & $-0.2474(2)$ & $0.52795(12)$ & $0.0395(4)$ \\
O5 & $0.9048(3)$ & $0.2533(2)$ & $0.67033(13)$ & $0.0420(4)$ \\
O6 & $0.8263(4)$ & $0.4872(3)$ & $0.5877(2)$ & $0.0657(7)$ \\
C1 & $0.7258(3)$ & $-0.1579(3)$ & $0.48041(18)$ & $0.0352(5)$ \\
C2 & $0.5601(3)$ & $-0.2465(4)$ & $0.4674(3)$ & $0.0525(8)$ \\
C3 & $1.1448(3)$ & $-0.2293(3)$ & $0.61239(16)$ & $0.0339(5)$ \\
C4 & $1.2395(4)$ & $-0.3641(4)$ & $0.6742(2)$ & $0.0548(7)$ \\
C5 & $0.8601(4)$ & $0.4018(4)$ & $0.6637(2)$ & $0.0460(7)$ \\
C6 & $0.8356(9)$ & $0.5060(6)$ & $0.7427(4)$ & $0.102(2)$
\end{tabular}


Table S3. Anisotropic Parameters for $\left[\mathrm{Cu}(\mathrm{OAc})_{2}\right]_{2} \cdot 2 \mathrm{AcOH}(2 \mathrm{a} \cdot \mathrm{AcOH})$.

\begin{tabular}{lllllll}
\hline atom & $U_{11}$ & $U_{22}$ & $U_{33}$ & $U_{23}$ & $U_{13}$ & $U_{12}$ \\
\hline Cu1 & $0.0336(2)$ & $0.0265(2)$ & $0.0260(2)$ & $-0.00281(8)$ & $0.00807(14)$ & $0.00223(8)$ \\
O1 & $0.0391(9)$ & $0.0422(9)$ & $0.0528(12)$ & $-0.0067(9)$ & $0.0185(8)$ & $-0.0034(7)$ \\
O2 & $0.0387(8)$ & $0.0415(10)$ & $0.0462(10)$ & $-0.0089(8)$ & $0.0094(8)$ & $-0.0072(7)$ \\
O3 & $0.0600(12)$ & $0.0379(9)$ & $0.0287(9)$ & $0.0011(7)$ & $0.0041(8)$ & $0.0081(8)$ \\
O4 & $0.0527(10)$ & $0.0344(9)$ & $0.0302(9)$ & $0.0035(7)$ & $0.0065(7)$ & $0.0104(7)$ \\
O5 & $0.0561(10)$ & $0.0399(10)$ & $0.0338(9)$ & $-0.0040(7)$ & $0.0183(8)$ & $0.0046(8)$ \\
O6 & $0.099(2)$ & $0.0403(12)$ & $0.0602(15)$ & $0.0024(11)$ & $0.0240(14)$ & $0.0222(12)$ \\
C1 & $0.0290(9)$ & $0.0280(11)$ & $0.0473(13)$ & $0.0047(9)$ & $0.0055(9)$ & $0.0003(8)$ \\
C2 & $0.0304(11)$ & $0.0422(14)$ & $0.084(2)$ & $0.0017(14)$ & $0.0100(13)$ & $-0.0040(10)$ \\
C3 & $0.0355(10)$ & $0.0322(11)$ & $0.0299(11)$ & $0.0047(8)$ & $-0.0015(9)$ & $-0.0009(8)$ \\
C4 & $0.0630(17)$ & $0.0449(15)$ & $0.0468(16)$ & $0.0124(13)$ & $-0.0083(13)$ & $0.0104(14)$ \\
C5 & $0.0561(16)$ & $0.0385(13)$ & $0.0494(17)$ & $-0.0104(11)$ & $0.0243(13)$ & $0.0030(11)$ \\
C6 & $0.179(6)$ & $0.062(2)$ & $0.086(3)$ & $-0.022(2)$ & $0.072(4)$ & $0.019(3)$
\end{tabular}


Table S4. Bond Lengths $(\AA)$ for $\left[\mathrm{Cu}(\mathrm{OAc})_{2}\right]_{2} \cdot 2 \mathrm{AcOH}(2 \mathrm{a} \cdot \mathrm{AcOH})$.

\begin{tabular}{llll}
\hline atoms & $\AA$ & atoms & $\AA$ \\
\hline Cu1-O2 & $1.952(2)$ & Cu1-O1 & $1.956(2)$ \\
Cu1-O3 & $1.962(2)$ & Cu1-O4 & $2.0036(19)$ \\
Cu1-O5 & $2.193(2)$ & Cu1-Cu1 & $2.5804(14)$ \\
O1-C1 & $1.262(3)$ & O2-C1 & $1.256(3)$ \\
O3-C3 & $1.243(3)$ & O4-C3 & $1.273(3)$ \\
O5-C5 & $1.210(3)$ & O6-C5 & $1.305(4)$ \\
C1-C2 & $1.501(3)$ & C3-C4 & $1.503(3)$ \\
C5-C6 & $1.497(5)$ & &
\end{tabular}


Table S5. Bond Angles (deg) for $\left[\mathrm{Cu}(\mathrm{OAc})_{2}\right]_{2} \cdot 2 \mathrm{AcOH}(\mathbf{2 a} \cdot \mathrm{AcOH})$.

\begin{tabular}{llll}
\hline atoms & deg & atoms & deg \\
\hline O2-Cu1-O1 & $169.79(8)$ & O2-Cu1-O3 & $89.30(10)$ \\
O1-Cu1-O3 & $89.96(11)$ & O2-Cu1-O4 & $89.50(9)$ \\
O1-Cu1-O4 & $89.39(10)$ & O3-Cu1-O4 & $169.57(8)$ \\
O2-Cu1-O5 & $93.48(8)$ & O1-Cu1-O5 & $96.66(9)$ \\
O3-Cu1-O5 & $101.22(9)$ & O4-Cu1-O5 & $89.20(9)$ \\
O2-Cu1-Cu1 & $85.23(6)$ & O1-Cu1-Cu1 & $84.57(7)$ \\
O3-Cu1-Cu1 & $88.07(8)$ & O4-Cu1-Cu1 & $81.50(7)$ \\
O5-Cu1-Cu1 & $170.61(5)$ & C1-O1-Cu1 & $122.81(17)$ \\
C1-O2-Cu1 & $122.40(17)$ & C3-O3-Cu1 & $120.98(17)$ \\
C3-O4-Cu1 & $125.77(16)$ & C5-O5-Cu1 & $129.9(2)$ \\
O2-C1-O1 & $125.0(2)$ & O2-C1-C2 & $117.7(2)$ \\
O1-C1-C2 & $117.3(2)$ & O3-C3-O4 & $123.7(2)$ \\
O3-C3-C4 & $119.2(2)$ & O4-C3-C4 & $117.2(2)$ \\
O5-C5-O6 & $124.1(3)$ & O5-C5-C6 & $122.9(4)$ \\
O6-C5-C6 & $113.0(3)$ & &
\end{tabular}


Table S6. Dihedral Angles (deg) for $\left[\mathrm{Cu}(\mathrm{OAc})_{2}\right] 2 \cdot 2 \mathrm{AcOH}(\mathbf{2 a} \cdot \mathrm{AcOH})$.

\begin{tabular}{llll}
\hline atoms & deg & atoms & deg \\
\hline Cu1-O2-C1-O1 & $-0.3(4)$ & Cu1-O2-C1-C2 & $178.04(18)$ \\
Cu1-O1-C1-O2 & $0.9(4)$ & Cu1-O1-C1-C2 & $-177.49(19)$ \\
Cu1-O3-C3-O4 & $-1.4(4)$ & Cu1-O3-C3-C4 & $176.6(2)$ \\
Cu1-O4-C3-O3 & $1.9(4)$ & Cu1-O4-C3-C4 & $-176.09(19)$ \\
Cu1-O5-C5-O6 & $6.8(5)$ & Cu1-O5-C5-C6 & $-174.1(3)$
\end{tabular}




\section{FEFF fitting analysis of $\mathrm{Cu}$ species in the reaction mixture of $\left[\mathrm{Pd}(\mathrm{OAc})_{2}(\right.$ phen $\left.)\right]$}

$(1 \mathrm{~b}) / \mathrm{Cu}(\mathrm{OAc})_{2} \cdot \mathrm{H}_{2} \mathrm{O}\left(2 \mathrm{a} \cdot \mathrm{H}_{2} \mathrm{O}\right)$.

For FEFF fitting analysis of $\mathrm{Cu}$ species in the reaction mixture of $\left[\operatorname{Pd}(\mathrm{OAc})_{2}(\right.$ phen $\left.)\right]$ $(\mathbf{1 b}) / \mathrm{Cu}(\mathrm{OAc})_{2} \cdot \mathrm{H}_{2} \mathrm{O}\left(\mathbf{2} \mathbf{a} \cdot \mathrm{H}_{2} \mathrm{O}\right)$ in dimethyl phtalate, the atomic coordinates obtained from single crystal X-ray structure of $\left[\mathrm{Cu}(\mathrm{OAc})_{2}\right] \cdot 2 \mathrm{H}_{2} \mathrm{O}\left(6 \mathbf{6} \cdot 2 \mathrm{H}_{2} \mathrm{O}\right)$, DFT-optimized $6 \mathbf{a} \cdot 2 \mathrm{H}_{2} \mathrm{O}$, and $2 \mathbf{a} \cdot \mathrm{AcOH}$ were used (Figures S4-S6). For the DFT-based geometry optimizations of $6 \mathrm{a} \cdot 2 \mathrm{H}_{2} \mathrm{O}$, the initial geometries were taken from the crystallographic structure and the calculation was performed at UB3LYP/6$31 \mathrm{G}(\mathrm{d}, \mathrm{p})$ level by using Gaussian 16 program. The atomic coordinate of DFT-optimized geometry of 6a. $2 \mathrm{H}_{2} \mathrm{O}$ was summarized in Table $\mathrm{S} 4$.
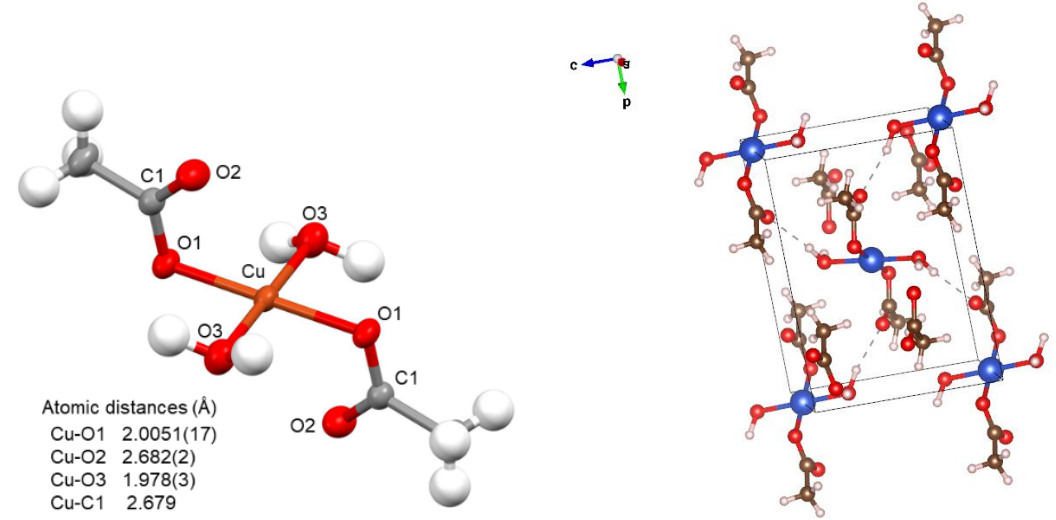

Figure S4. The single crystal X-ray structure of $6 \mathrm{a} \cdot 2 \mathrm{H}_{2} \mathrm{O}$ (ORTEP drawing).
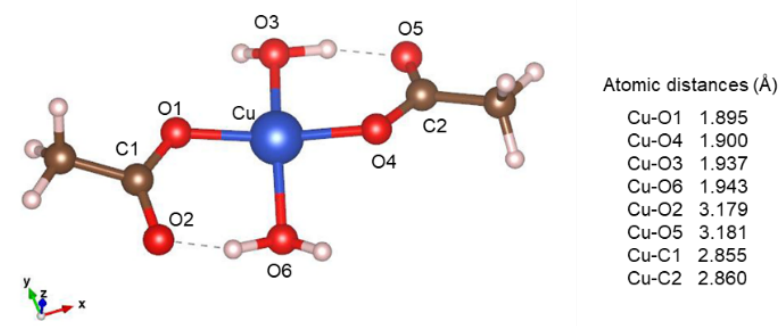

Figure S5. DFT-optimized molecular geometry of $6 \mathrm{a} \cdot 2 \mathrm{H}_{2} \mathrm{O}$ using the single crystal X-ray structure of $6 \mathbf{a} \cdot 2 \mathrm{H}_{2} \mathrm{O}$ as the initial geometry. The molecular geometry optimization was performed at UB3LYP/6$31 G(d, p)$ level of theory.

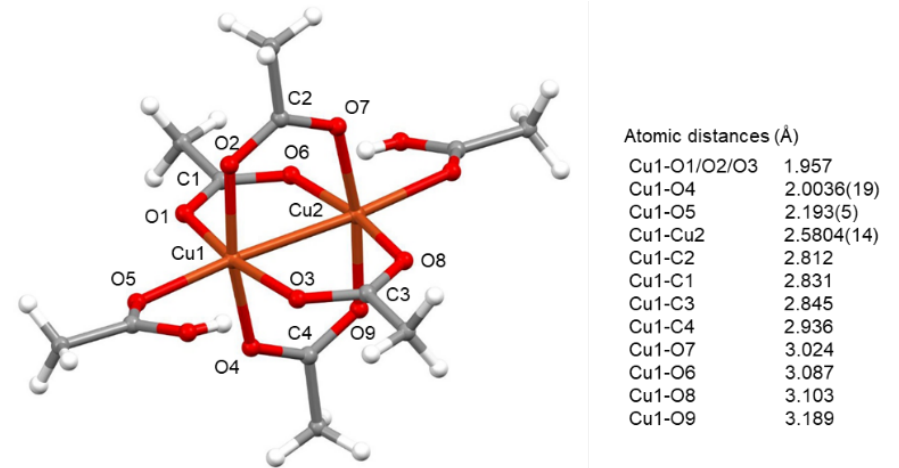

Figure S6. The single crystal X-ray structure of 2a·AcOH (ORTEP drawing). 
Table S7. Atomic coordinate of the molecular geometry of DFT-optimized $\mathbf{6 a} \cdot 2 \mathrm{H}_{2} \mathrm{O}$ in Figure S5.

\begin{tabular}{|c|c|c|c|}
\hline & \multicolumn{3}{|c|}{ A tom ic Coord inate } \\
\hline Sym bol & $\mathrm{x}$ & $\mathrm{y}$ & $\mathrm{z}$ \\
\hline Cu1 & -0.012 & 0.0715 & -0.0719 \\
\hline 01 & -0.5212 & -1.6819 & -0.7195 \\
\hline 02 & 1.6997 & -0.611 & 0.3892 \\
\hline C & 2.8368 & -0.0466 & 0.1478 \\
\hline C & 4.0557 & -0.8711 & 0.5042 \\
\hline H 1 & 4.9684 & -0.3249 & 0.2671 \\
\hline H 2 & 4.0336 & -1.8183 & -0.0426 \\
\hline H 3 & 4.0326 & -1.1128 & 1.571 \\
\hline 03 & 2.985 & 1.0968 & -0.3391 \\
\hline H 4 & -0.0189 & -2.4028 & -0.3183 \\
\hline 04 & -1.7996 & 0.6722 & 0.1188 \\
\hline C 1 & -2.8551 & -0.0701 & 0.1442 \\
\hline C 2 & -4.1431 & 0.6525 & 0.4768 \\
\hline H5 & -4.9768 & -0.0485 & 0.5114 \\
\hline H 6 & -4.0404 & 1.1647 & 1.4376 \\
\hline H 7 & -4.3346 & 1.4201 & -0.279 \\
\hline 05 & -2.8817 & -1.3017 & -0.0856 \\
\hline H 8 & -1.5331 & -1.7295 & -0.4774 \\
\hline 06 & 0.6385 & 1.8954 & -0.2325 \\
\hline H 9 & 0.2318 & 2.4721 & -0.8894 \\
\hline H 10 & 1.6527 & 1.7612 & -0.3841 \\
\hline & & & \\
\hline
\end{tabular}

The corresponding XAS data were processed using Athena by extracting the EXAFS oscillations $\chi(k)$ as a function of photoelectron wave number $k$. Fourier transformation of the $k^{3}$ weighted $\chi$ from $k$ space to $r$ space was carried out to obtain the radial distribution function. The EXAFS fitting calculation was performed by FEFF $6{ }^{1}$ program embedded with Artemis, where the theoretical scattering paths were generated from crystallographic coordinate or DFT-optimized Xray structure as shown in Figures S4-S6. The parameters for FEFF fitting analysis are as follows: $\mathrm{Ab}$-Sc: the X-ray absorbing atom and the scattering atom; $\mathrm{CN}$ : coordination number; $D W$ : DebyeWaller factor $\left(\AA^{2}\right) ; \Delta E$ : energy shift (eV); $R$ : atomic distance $(\AA)$. The parameter for the many-body effect of $S_{0}{ }^{2}$ is fixed to 1.0 for all fitting calculations.

For fitting calculation of $6 \mathrm{a} \cdot 2 \mathrm{H}_{2} \mathrm{O}$ based on X-ray structure, eight independent parameters (CuO1/O3: $\left.S_{0}^{2}, e 1, r 1, \sigma 1 ; \mathrm{Cu}-\mathrm{C} 1, \mathrm{Cu}-\mathrm{O} 2: \mathrm{S}_{0}^{2}, \mathrm{e} 2, \mathrm{r} 2, \sigma 2\right)$ with three scattering paths (1st shell: $\mathrm{Cu}-$ $\mathrm{O} 1 / \mathrm{O} 3,2$ nd shell: $\mathrm{Cu}-\mathrm{C} 1, \mathrm{Cu}-\mathrm{O} 2)$ give the fitting results with adequate accuracy as shown in Figures S7, where the many-body effect $S_{0}{ }^{2}$ parameter was fixed with an appropriate value as $S_{0}{ }^{2}=$ 1.0 .

For fitting calculation of $6 \mathrm{a} \cdot 2 \mathrm{H}_{2} \mathrm{O}$ based DFT-optimized structure, eight independent parameters (Cu-O1/O4, Cu-O3/O6: $\mathrm{S}_{0}{ }^{2}, \mathrm{e} 1, \mathrm{r} 1, \sigma 1$; $\left.\mathrm{Cu}-\mathrm{C} 1 / \mathrm{C} 2, \mathrm{Cu}-\mathrm{O} 2 / 5: \mathrm{S}_{0}{ }^{2}, \mathrm{e} 2, \mathrm{r} 2, \sigma 2\right)$ with four scattering paths (1st shell: $\mathrm{Cu}-\mathrm{O} 1 / \mathrm{O} 4, \mathrm{Cu}-\mathrm{O} 3 / \mathrm{O} 6,2$ nd shell: $\mathrm{Cu}-\mathrm{C} 1 / \mathrm{C} 2, \mathrm{Cu}-\mathrm{O} 2 / 5)$ give the fitting results with adequate accuracy as shown in Figures $S 8$, where the many-body effect $S_{0}{ }^{2}$ parameter was fixed with an appropriate value as $S_{0}{ }^{2}=1.0$. The refined parameters were summarized in tables with each fitting result. 
For fitting calculation of $\mathbf{2 a} \cdot \mathbf{A c O H}$ based on X-ray structure, nine independent parameters (Cu1-O1/O2/3, Cu1-O4, Cu1-O5: $S_{0}^{2}, \mathrm{e} 1, \mathrm{r} 1$, o1; Cu1-Cu2: $S_{0}^{2}$, e1, r2, o1; Cu1-C1/C2, Cu1-C3, Cu1-C4, Cu1-O-C, Cu1-O7, Cu1-06/O8, Cu1-O9: $S_{0}^{2}$, e2, r3, o2) with eleven scattering paths (1st shell: Cu1-O1/O2/3, Cu1-O4, Cu1-O5, 2nd shell: Cu1-Cu2, 3rd shell: Cu1-C1/C2, Cu1-C3, Cu1-C4, Cu1-O-C, Cu1-07, Cu1-06/O8, Cu1-09) give the fitting results with adequate accuracy as shown in Figures S6, where the many-body effect $S_{0}{ }^{2}$ parameter was fixed with an appropriate value as $S_{0}{ }^{2}=$ 1.0 .

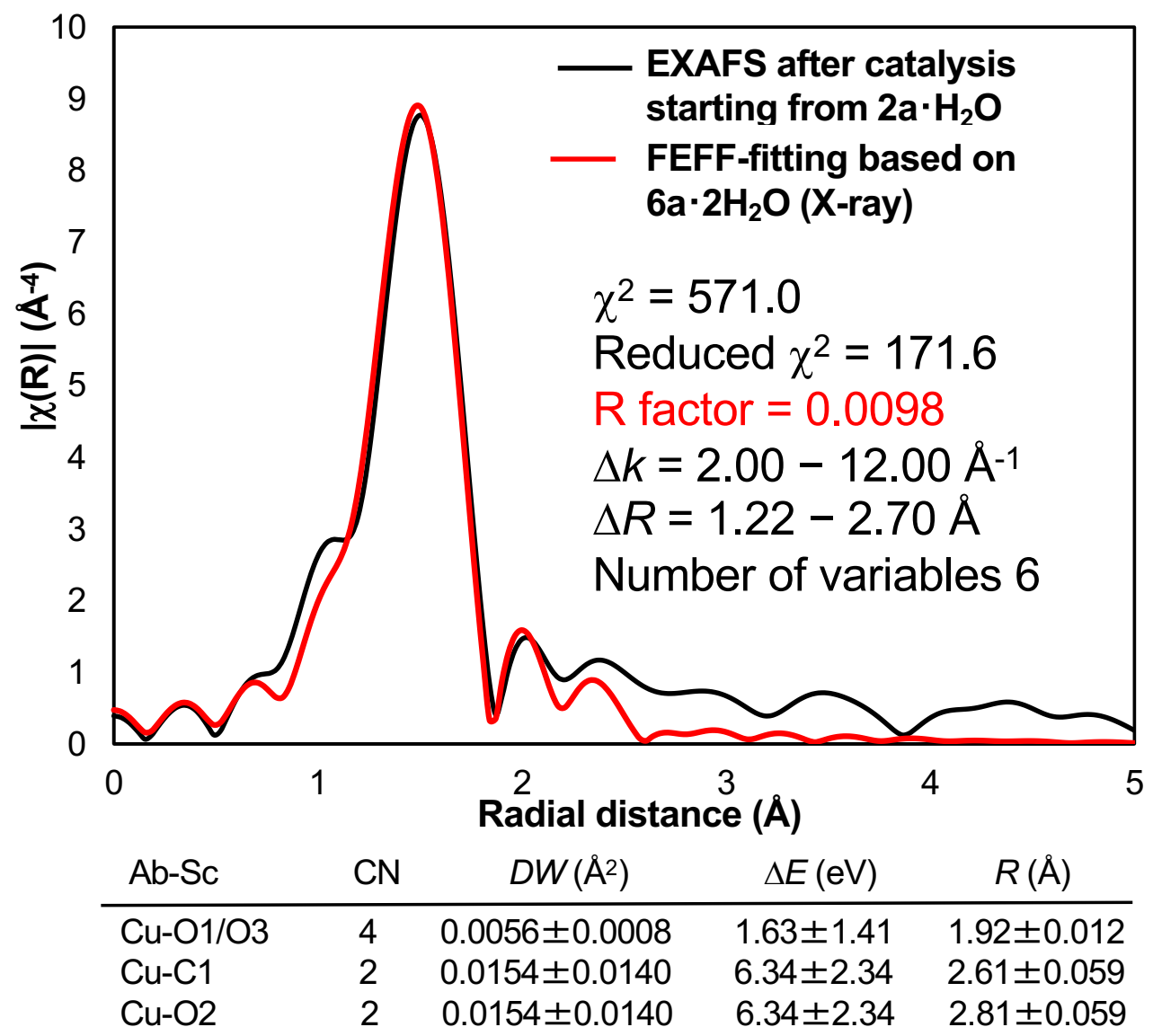

Figure S7. FEFF fitting analysis on the EXAFS spectrum after catalysis starting from $\left[\mathrm{Pd}(\mathrm{OAc})_{2}(\mathrm{phen})\right]$ $(1 \mathbf{b}) / \mathrm{Cu}(\mathrm{OAc})_{2} \cdot \mathrm{H}_{2} \mathrm{O}\left(2 \mathrm{a} \cdot \mathrm{H}_{2} \mathrm{O}\right)$ using the CIF-based model of $6 \mathrm{a} \cdot 2 \mathrm{H}_{2} \mathrm{O}$ shown in Figure $\mathrm{S} 4$. The bottom table shows a summary of selected FEFF parameters: Ab-Sc: absorbing and scattering atoms for shell, $\mathrm{CN}$ : coordination number, DW: Debye-Waller factor, $\Delta \mathrm{E}$ : initial bond length for scattering path based on single crystal X-ray structure analysis, R: bond length from FEFF fitting. 


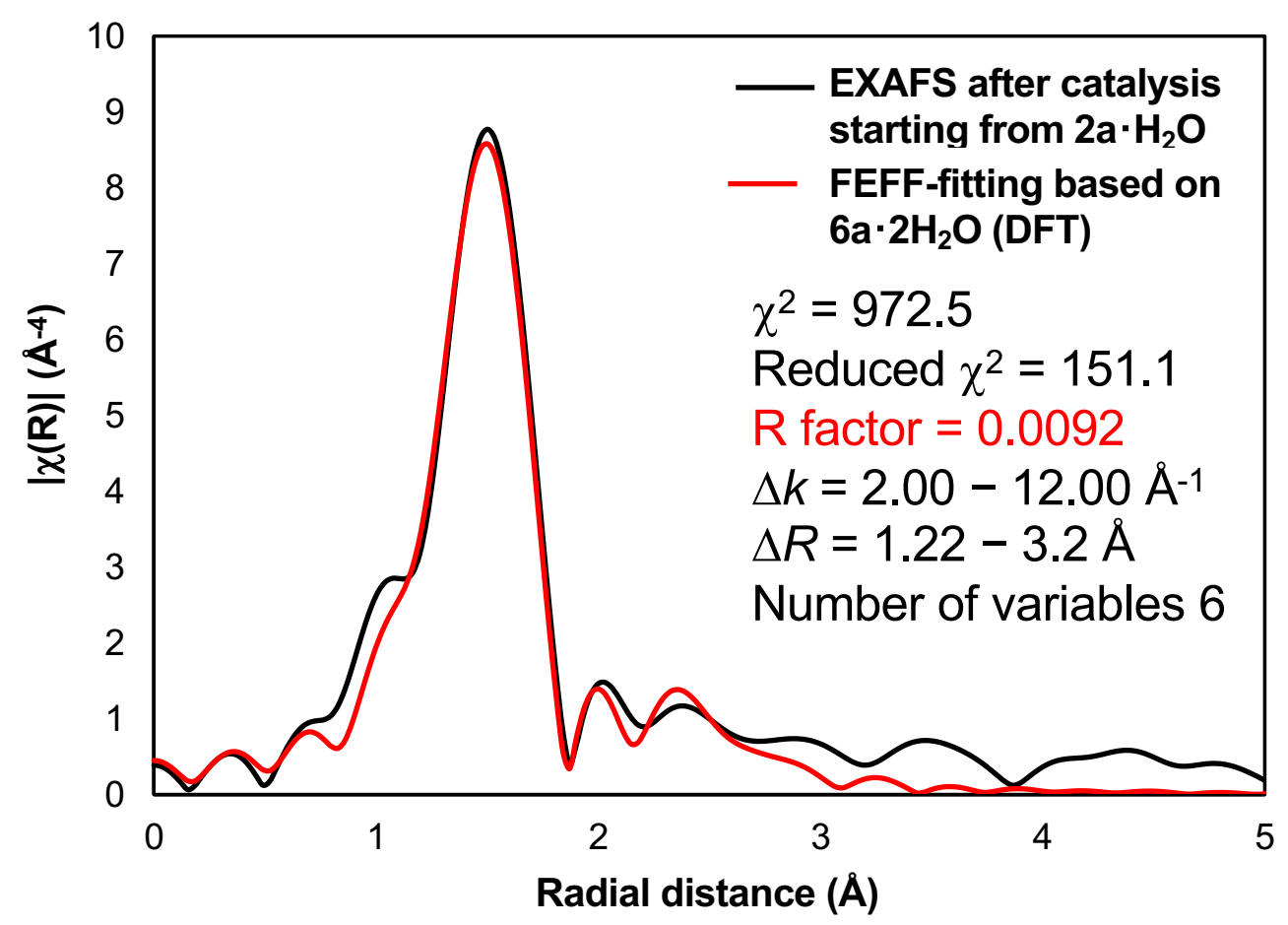

\begin{tabular}{llccc}
$\mathrm{Ab}-\mathrm{Sc}$ & $\mathrm{CN}$ & $D W\left(\AA^{2}\right)$ & $\Delta E(\mathrm{eV})$ & $R(\AA)$ \\
\hline $\mathrm{Cu}-\mathrm{O} 1 / \mathrm{O} 4$ & 2 & $0.0055 \pm 0.0008$ & $4.23 \pm 0.90$ & $1.91 \pm 0.094$ \\
$\mathrm{Cu}-\mathrm{O} 3 / \mathrm{O} 6$ & 2 & $0.0055 \pm 0.0008$ & $4.23 \pm 0.90$ & $1.95 \pm 0.094$ \\
$\mathrm{Cu}-\mathrm{C} 1 / \mathrm{C} 2$ & 2 & $0.0085 \pm 0.0050$ & $-2.87 \pm 10.98$ & $2.82 \pm 0.091$ \\
$\mathrm{Cu}-02 / \mathrm{O} 5$ & 2 & $0.0085 \pm 0.0050$ & $-2.87 \pm 10.98$ & $3.14 \pm 0.091$
\end{tabular}

Figure S8. FEFF fitting analysis on the EXAFS spectrum after catalysis starting from $\left[\mathrm{Pd}(\mathrm{OAc})_{2}(\mathrm{phen})\right]$ $(\mathbf{1} \mathbf{b}) / \mathrm{Cu}(\mathrm{OAc})_{2} \cdot \mathrm{H}_{2} \mathrm{O}\left(\mathbf{2} \mathbf{a} \cdot \mathrm{H}_{2} \mathrm{O}\right)$ using the DFT-optimized model of $6 \mathbf{a} \cdot 2 \mathrm{H}_{2} \mathrm{O}$ shown in Figure S5. The bottom table shows a summary of selected FEFF parameters: Ab-Sc: absorbing and scattering atoms for shell, $\mathrm{CN}$ : coordination number, DW: Debye-Waller factor, $\triangle \mathrm{E}$ : initial bond length for scattering path based on DFT, R: bond length from FEFF fitting. 


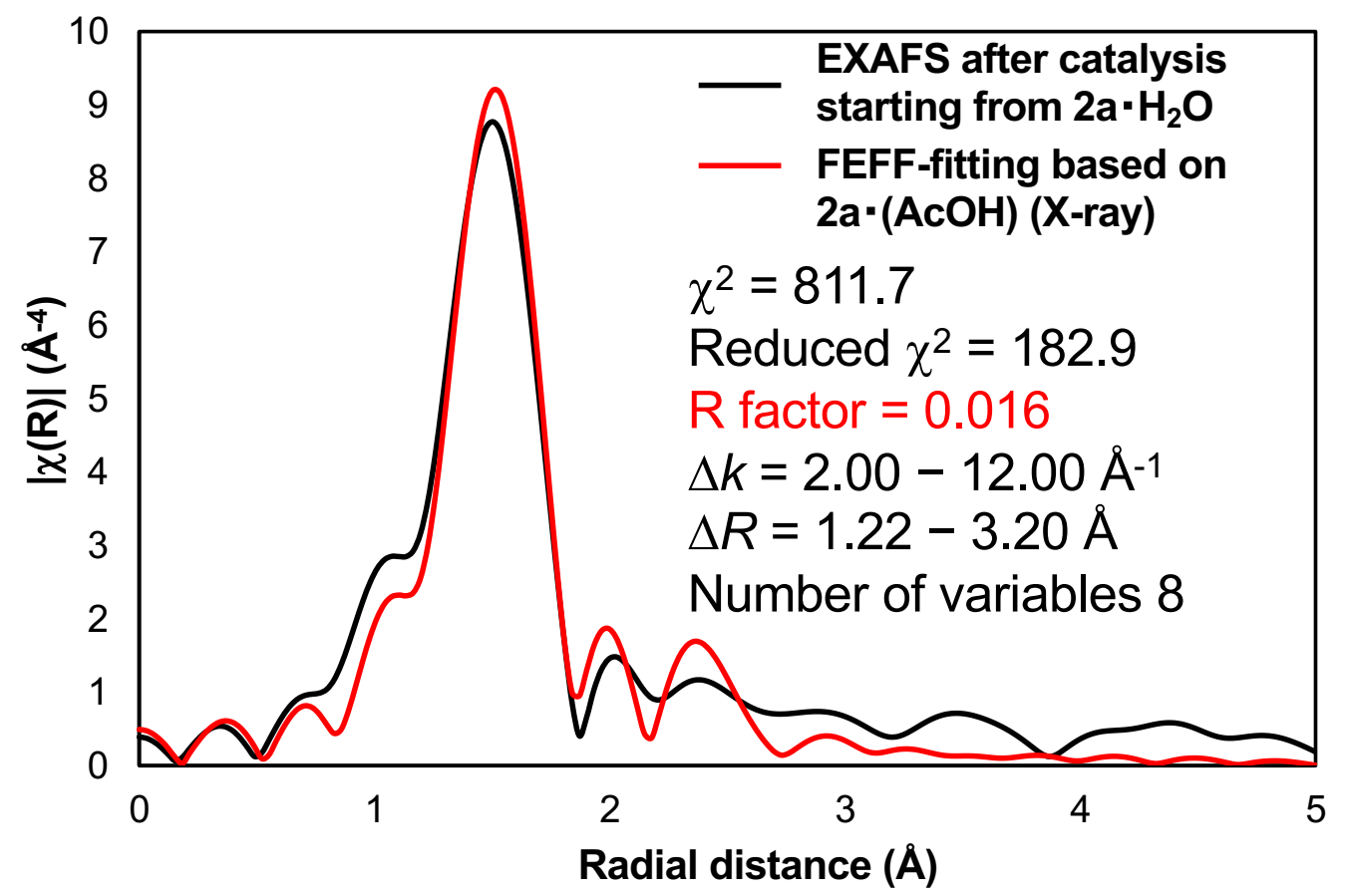

\begin{tabular}{lcccc} 
Ab-Sc & $C N$ & $D W\left(\AA^{2}\right)$ & $\Delta E(\mathrm{eV})$ & $R(\AA)$ \\
\hline Cu1-O1/O2/O3 & 3 & $0.0037 \pm 0.0007$ & $7.61 \pm 1.22$ & $1.94 \pm 0.011$ \\
Cu1-O4 & 1 & $0.0037 \pm 0.0007$ & $7.61 \pm 1.22$ & $1.99 \pm 0.011$ \\
Cu1-O5 & 1 & $0.0037 \pm 0.0007$ & $7.61 \pm 1.22$ & $2.17 \pm 0.011$ \\
Cu1-Cu2 & 1 & $0.0118 \pm 0.0077$ & $7.61 \pm 1.22$ & $2.44 \pm 0.059$ \\
Cu1-C1/C2 & 2 & $0.0136 \pm 0.0083$ & $-0.84 \pm 5.83$ & $2.85 \pm 0.108$ \\
Cu1-C3 & 1 & $0.0136 \pm 0.0083$ & $-0.84 \pm 5.83$ & $2.88 \pm 0.108$ \\
Cu1-C4 & 1 & $0.0136 \pm 0.0083$ & $-0.84 \pm 5.83$ & $2.97 \pm 0.108$ \\
Cu1-O-C & 6 & $0.0136 \pm 0.0083$ & $-0.84 \pm 5.83$ & $3.05 \pm 0.108$ \\
Cu1-O7 & 1 & $0.0136 \pm 0.0083$ & $-0.84 \pm 5.83$ & $3.05 \pm 0.108$ \\
Cu1-O6/O8 & 2 & $0.0136 \pm 0.0083$ & $-0.84 \pm 5.83$ & $3.09 \pm 0.108$ \\
Cu1-O9 & 1 & $0.0136 \pm 0.0083$ & $-0.84 \pm 5.83$ & $3.19 \pm 0.108$
\end{tabular}

Figure S9. FEFF fitting analysis on the EXAFS spectrum after catalysis starting from $\left[\mathrm{Pd}(\mathrm{OAc})_{2}(\mathrm{phen})\right]$ $(\mathbf{1} \mathbf{b}) / \mathrm{Cu}(\mathrm{OAc})_{2} \cdot \mathrm{H}_{2} \mathrm{O}\left(\mathbf{2} \mathbf{a} \cdot \mathrm{H}_{2} \mathrm{O}\right)$ using the DFT-optimized model of $\mathbf{2 a} \cdot \mathrm{AcOH}$ shown in Figure S6. The bottom table shows a summary of selected FEFF parameters: Ab-Sc: absorbing and scattering atoms for shell, $\mathrm{CN}$ : coordination number, DW: Debye-Waller factor, $\triangle \mathrm{E}$ : initial bond length for scattering path based on single crystal $X$-ray structure analysis, $R$ : bond length from FEFF fitting. 